\title{
A Rare Anomaly of the Long Head of the Biceps Brachii Muscle
}

\author{
Kumar $\mathbf{S}^{1^{*}}$, Baidya $\mathbf{R}^{1}$, Baral $\mathbf{P}^{2}$ \\ ${ }^{1}$ Assistant professor of Anatomy teaching in Radiology division of \\ gross anatomy and anatomical body visualization, Weill Cornell Medicine, New York \\ ${ }^{2}$ Professor, Anatomy Department, Gandaki Medical College \& Teaching Hospital, Pokhara, Nepal
}

\author{
Keywords \\ Arm, Biceps brachii, \\ Glenoid, Long head, Muscle.

\section{Corresponding author} \\ *Dr. Sushil Kumar \\ Assistant professor of \\ Anatomy teaching in \\ Radiology Division of Gross Anatomy and \\ anatomical body visualization \\ Weill Cornell Medicine, New York \\ Email:szk2008@med.cornell.edu
}

\begin{abstract}
Introduction: Biceps brachii is a muscle of arm which brings about supination when fore-arm is flexed and flexion of elbow joint. Proximally it is attached with two heads: long and short heads.

Case report: The absence of long head of biceps brachii muscle is very rare anomaly. It may be unilateral or bilateral with or without other congenital anomalies. The exact prevalence of this anomaly is unknown. This anomaly has been reported to occur as the result of an insult to the fetus during the sixth or seventh week of gestation, at which time the long head of the biceps tendon is developing.
\end{abstract}

\section{INTRODUCTION}

The bicep brachii muscle has two tendinous origins. The short head arises by a thick, flattened tendon from coracoid process of scapula with coracobrachialis. The long head starts within the capsule of the shoulder joint as a long narrow tendon, running from the supraglenoid tubercle of the scapula at the apex of the glenoid cavity, where it is continuous with glenoid labrum. The tendon of the long head arched over the humeral head, emerges from the capsule under the transverse humeral ligament, and descends in the intertubercular sulcus. The two tendons lead into elongated bellies which turn into flattened tendon at elbow joint which is attached to the rough posterior area of the radial tuberosity ${ }^{1}$.

\section{CASE REPORT}

During a routine anatomical dissection of a 45 year old female cadaver at the Department of Anatomy, at Weill Cornell Medicine, we observed an intriguing finding. In left arm long head of biceps brachii tendon was absent. The short head of biceps brachii was normal and was coming from tip of the coracoid process along with the coracobrachialis. Distally it was attached on the posterior aspect of radial tuberosity. It was innervated by musculocutaneous nerve. On right arm both head had normal development. We did not observe any other variations.

Fig 1: Showing absence of the long head of bicep brachii

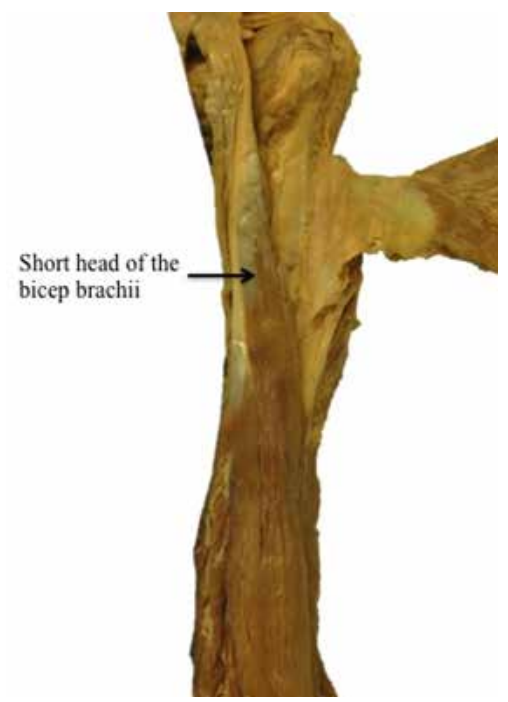




\section{DISCUSSION}

Anomalies of the long head of the bicep brachii include absence, hypoplasia, duplication and various origins (from the capsular ligaments, the bicipital groove, the insertion of the oracobrachialis, the tendon of the pectoralis major and the greater tuberosity of the humerus) $)^{2}$. Absence of the tendon of the long head of the biceps brachii can be congenital and is associated with other anomalies such as the VATER association, which includes vertebral defects, anal atresia, tracheo-esophageal fistula with esophageal atresia, radial anomalies and renal anomalies ${ }^{3}$. Variations, with the exception of congenitally absent long heads are classified in terms of their relationship, or extent of fusion, with the supraspinatus tendon ${ }^{4}$.

Wahl and McGillivray classified the anatomical variants of the intraarticular segment of the tendon of the long head of biceps brachii on the basis of arthroscopy into four types; incomplete proximal mesenteric, incomplete distal mesenteric, complete mesenteric, and congenitally absent ${ }^{5}$. Diericks et al analyzed 2976 shoulder pathologies and classified the 57 shoulders (1.91\%) that had an anatomic variation into four types: Mesotendon, adherent, bifurcated, and congenitally absent ${ }^{6}$. These attempts to classify the many variations of the biceps brachii demonstrate the great variety of anatomical anomalies that are associated with this muscle.

Variations of the long head of biceps brachii could be found accidentally during the treatment of the various shoulder disorders including cuff degeneration, shoulder impingement, acromioclavicular joint arthritis ${ }^{7,8}$. Long head of biceps brachii anomalies have been reported as a possible mechanism for development of shoulder instability 9

In the clinical settings, most of the anatomical variations of the long head of biceps brachii are not diagnosed during radiological assessments, but rather during arthroscopic inspection. This underlines the need for surgical awareness during arthroscopic surgery due to possible unexpected anatomical variations or lesions of the $\mathrm{LHB}^{4}$. Diagnostic difficulties at the time of shoulder arthroscopy also arise from the fact that the tendon of the long head of biceps brachii is used as a landmark and may confuse even experienced surgeons $\mathbf{s}^{3,9}$.

\section{CONCLUSION}

In conclusion, the present case reports describe a rare anomaly of the biceps brachii. Although rarely encoun- tered, it can create diagnostic difficulties, as it is not easily discovered through routinely used imaging modalities. Thus, clinical awareness of their existence and MRI appearance can help prevent misdiagnosis and avoid unnecessary surgery.

\section{REFERENCES}

1. Standaring S. Gray's anatomy. $41^{\text {st }}$ edition Elsevier. 2016: 824.

2. Georgi P Georgiev, Jelev L. Bilateral hypoplasia of the long head of the biceps brachii muscle. International journal shoulder surgery. 2011; 5: 26-27.

3. Gillardin P, Vanhoenacker FM, Wauters T, De Backer AI. Congenital absence of long head of the biceps tendon. JBR-BTR. 2013; 96; 320.

4. Kwak S, Lee S, Song BW, Lee M, Suh K. Three cases of rare anatomic variations of the long head of the biceps brachii. CiSE. 2015; 18; 96-101.

5. Wahl CJ, MacGillivray JD. Three congenital variations in the long head of the biceps tendon: a review of pathoanatomic considerations and case reports. J Shoulder Elbow Surg. 2007; 16; 25-30.

6. Dierickx C, Ceccarelli E, Conti M, Vanlommel J, Castagna A. Variations of the intra-articular portion of the long head of the biceps tendon: A classification of embryologically explained variations. J Shoulder Elbow Surg. 2009; 18: 556-565.

7. Dierickx C, Ceccarelli E, Conti M, Vanlommel J, Castagna A. Variations of the intra-articular portion of the long head of the biceps tendon: A classification of embryologically explained variations. J Shoulder Elbow Surg. 2009; 18: 556-565.

8. Audenaert EA, Barbaix EJ, Van Hoonacker P, Berghs BM. Extraarticular variants of the long head of the biceps brachii: A reminder of embryology. J Shoulder Elbow Surg. 2008; 17: 114-117.

9. Gaskin CM, Golish SR, Blount KJ, Diduch DR. Anomalies of the long head of the biceps brachii tendon: Clinical significance, MR arthrographic findings, and arthroscopic correlation in two patients. Skeletal Radiol. 2007; 36: 785- 789. 